\title{
Rice Tolerance to Drought is Complex Both Physiologically and Genetically
}

Paula Jernigan, Luca Nies, George Fernandes, Roberto Quesada

Microbiological and Enzyme Chemistry Research Unit, National Research Centre, Dokki, Cairo Institute of Oceanography and Fisheries, Kayed Bey, Alexandria, Egypt

This paper has been withdrawn by bioRxiv because its content, including the author names, was fabricated and fraudulently submitted in what may have been an attempt to game citation statistics or other metrics. 